\title{
Orion GNC Mitigation Efforts for Van Allen Radiation
}

\author{
Ellis T. King ${ }^{1}$ and Mark Jackson ${ }^{2}$ \\ Charles Stark Draper Laboratory, Houston, TX, 77058
}

The Orion Crew Module (CM) is NASA's next generation manned space vehicle, scheduled to return humans to lunar orbit in the coming decade. The Orion avionics and GN\&C architectures have progressed through a number of project phases and are nearing completion of a major milestone. The first unmanned test mission, dubbed "Exploration Flight Test One" (EFT-1) is scheduled to launch from NASA Kennedy Space Center late next year and provides the first integrated test of all the vehicle systems, avionics and software.

The EFT-1 mission will be an unmanned test flight that includes a high speed re-entry from an elliptical orbit, which will be launched on an expendable launch vehicle (ELV). The ELV will place CM and the ELV upper stage into a low Earth orbit (LEO) for one revolution. After the first LEO, the ELV upper stage will re-ignite and place the combined upper stage/CM into an elliptical orbit whose perigee results in a high energy entry to test CM response in a relatively high velocity, high heating environment. While not producing entry velocities as high as those experienced in returning from a lunar orbit, the trajectory was chosen to provide higher stresses on the thermal protection and guided entry systems, as compared against a lower energy LEO entry. However the required entry geometry with constraints on inclination and landing site result in a trajectory that lingers for many hours in the Van Allen radiation belts. This exposes the vehicle and avionics to much higher levels of high energy proton radiation than a typical LEO or lunar trajectory would encounter. As a result, Van Allen radiation poses a significant risk to the Orion avionics system, and particularly the Flight Control Module (FCM) computers that house the GN\&C flight software.

The measures taken by the Orion GN\&C, Flight Software and Avionics teams to mitigate the risks associated with the Van Allen radiation on EFT-1 are covered in the paper. Background on the Orion avionics subsystem is provided, as well as an overview of the GN\&C software architecture. The measures taken to handle radiation induced failure of the one or both of the FCM's are presented, and finally simulation and actual hardware-in-the-loop (HWIL) results are shown confirming the validity of the implementation.

The paper presents an overview of the Orion avionics architecture describing the GNC sensors, onboard data network as well as the flight control computers and their planned restart capabilities. GN\&C sensors include two Orion Inertial Measurement Units (OIMU's), a Vision Processing Unit (VPU) to process camera images, three barometric altimeters and a single GPS receiver. All of the sensors communicate to one of two Power and Data Units (PDU's). The PDU's multiplex analog and serial data from the sensors and write the data to the Orion Data Network (ODN). The OIMU's write measurement messages directly as onto the ODN, but they are routed through PDU network switches.

Sensor data are passed via the ODN to the GN\&C software application within one of two selfchecking pair Flight Control Modules (FCM's). Each self-checking FCM houses identical instances of the GN\&C application which executes the Orion Flight software, providing fault tolerance for the operating FCM hardware for a number of hardware upset events. FCM's may communicate between one another via a "cross channel" data connection on the ODN. Cross channel data serve to ensure synchronization of critical events as well as to provide restart data from an operating FCM to a nonoperating FCM.

\footnotetext{
${ }^{1}$ Senior Member of the Technical Staff, Charles Stark Draper Laboratory, 17629 El Camino Real, Suite 470, Houston, TX 77058, AIAA Senior Member.

${ }^{2}$ Principal Member of the Technical Staff, Charles Stark Draper Laboratory, 17629 El Camino Real, Suite 470, Houston, TX 77058, AIAA Senior Member.
} 
When radiation upset event conditions are detected by the self-checking pair FCM hardware (or if one is intentionally commanded by ground operators), this triggers a restart of the affected FCM hardware while the rest of the avionics continue to operate. In the event that the non-restarting FCM is operating and uninhibited it continues to fly the vehicle closed loop without interruption. However for EFT-1 there is also a real, although low probability chance that the secondary FCM may not be operational at the time of the restart. This constitutes what is referred to as a dual FCM restart and was deemed to be significant enough of a risk to the program to develop a solution in software. In the dual restart case a combination of data stored in non-volatile FCM memory and auxiliary VPU hardware is used to provide enough critical data to restart the FCM(s) without an active counterpart. In either restart case, all of the critical GN\&C flight software states and data must be transferred to the restarting FCM and utilized in the application software to initialize all the algorithms at the appropriate point in the mission event sequence. The paper presents a high level overview of the embedded restart logic on each FCM for determining upon restart or power up whether one of the restart cases is encountered or if the vehicle is proceeding through a nominal power up on the launch pad.

The GN\&C software executes on one of the many software partitions allocated on each FCM. This paper presents an overview of the EFT-1 GN\&C software architecture with a heavy focus on navigation, as most of the complexity in performing inflight restarts deals with restarting the navigation software. The timing and rate monotonic scheduling scheme is summarized to provide background for the sections that discuss how the multi-rate navigation system is re-anchored to the current Orion time during the restart process.

The Orion GN\&C software architecture includes a GN\&C command interface (GCI) that provides moding commands and configuration data to navigation, guidance and control domain modules. Automated sequences are enacted within GCI by sequencing through data configurable activities loaded onto the vehicle via Separately Loadable Databases (SLDBs). Each activity consists of the appropriate mode commands and configuration data needed to accomplish the activity objective. GCI also contains software to perform automated transitions between activities based on data configurable transition criteria. The vehicle "Timeline Manager (TM)" is responsible for coordinating event transitions across all vehicle subsystems including GN\&C, and the interface for communicating changes across all subsystems is through "mission segments". Within the GN\&C subsystem, GCI responds to a new mission segment by kicking off a new sequence of activities. Transitions between mission segments within the TM software are often based on state data and flags provided by GN\&C. While all of the GN\&C state data cannot be synchronized between the FCMs, transitions between the high level segments are synchronized to ensure that segment boundaries could never occur at slightly different times within each FCM. This critical event synchronization is accomplished by including the transition status of the counterpart FCM in the segment transition criteria. The paper describes the critical state data exchanged between TM and GCI counterpart instances in order to ensure that vehicle configuration parameters, sequencing information as well as command data are preserved during a restart.

The navigation software is naturally sensitive to initial conditions during an inflight restart. Only a subset of the navigation filter states can be provided to initialize navigation filters in the restarting FCM, and the specific solution determined in each FCM is naturally subject to many details including measurement processing, accept/reject counters and other nuances. Therefore the navigation software instances on each FCM will naturally produce slightly different solutions, although neither is more correct than the other. This aspect of the Orion design produces more complexity in the GN\&C system particularly when switching from one FCM as the active flight computer since the closed loop system will invariably have to tolerate slight transients as the process of switching FCMs completes. The paper discusses of the issues surrounding this complexity and the mitigation steps taken to ensure robustness for the EFT-1 and future missions.

The navigation software is divided in to two "channels" associated with each of the redundant Orion IMUs. Each channel includes both filtered and inertial solutions. The filtered solutions incorporate GPS measurements within a $1 \mathrm{~Hz}$ Extended Kalman Filter (EKF) that interacts with a $40 \mathrm{~Hz}$ inertial propagator. In addition a separate, inertial solution is maintained on the vehicle for each navigation 
channel to provide a backup for the filtered navigation solution taking measurements from the Orion GPS receiver which has never been flown in an exo-atmospheric environment. The paper discusses the mechanisms for initializing both the filtered and inertial states during a restart as well as the navigation trades used to identify and prioritize the subset of navigation filter states that passed between FCMs. Additionally other non-navigation algorithms also require cross channel initialization, including fault detection, isolation and recovery (FDIR) and entry guidance algorithms. Finally, after describing the software architecture and necessary navigation features required to support single and dual FCM restarts, the effect on the navigation system performance is quantified. This is achieved through several methods including analysis of the EFT-1 mission trajectory and nominal mission timeline, the navigation EKF implementation and parameterization, as well as Monte Carlo simulation results. In addition, operational considerations are covered as part of the study, including the implications of restarts upon telemetry and how the automated restart process may be monitored from the ground. Flight rules affected by FCM restarts are discussed, along with rules that govern the use of available ground commands such as a contingency navigation state update/replacement. Operational implications for future Orion missions are also touched upon. Ultimately, robustness to radiation induced inflight restarts is shown within the GN\&C system for a variety of stressing yet possible outcomes, to significantly increase probability of mission success for the EFT-1 mission. 